\title{
The effect of lens wear on refractive index of conventional hydrogel and silicone-hydrogel contact lenses: A comparative study
}

\author{
M. Lira ${ }^{\text {a,* }}$, L. Santos ${ }^{\text {b }}$ J. Azeredo ${ }^{\text {b }}$, E. Yebra-Pimentel ${ }^{\text {c }}$, \\ M.E.C.D. Real Oliveira ${ }^{\mathrm{a}}$

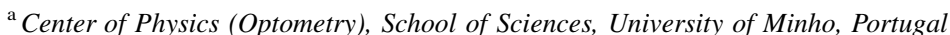 \\ ${ }^{\mathrm{b}}$ IBB-Institute for Biotechnology and Bioengineering, Center of Biological Engineering, University of Minho, Portugal \\ ${ }^{\mathrm{c}}$ School of Optics and Optometry, University of Santiago de Compostela, Spain
}

\begin{abstract}
Purpose: The purpose of this work was to evaluate the ability of four silicone-hydrogel contact lenses (galyfilcon A, balafilcon A, lotrafilcon A and lotrafilcon B) to retain their equilibrium water content before and after wear, through measurements of refractive index and compare with that of a conventional disposable hydrogel contact lens (etafilcon A).

Methods: The refractive indices of 115 contact lenses were measured using an automated refractometer (CLR 12-70, Index Instruments, Cambridge, U.K.) before and after a schedule of daily wear by 58 patients for 30 days in the case of silicone-hydrogel lenses and 15 days for the conventional contact lenses.

Results: In the silicone-hydrogel contact lenses the changes on the refractive indices were not statistically significant, however after being worn the refractive index of the conventional etalfilcon A hydrogel contact lens increased significantly $(p<0.001)$.

Conclusion: The results presented here show that after being worn the silicone-hydrogel contact lens, show more capacity to retain or to reach their initial equilibrium water content than conventional hydrogel contact lenses. This suggests that the silicone-hydrogel contact lenses are less susceptible to spoilation over time maintaining its biocompatibility and contributing to the clinical success of lens performance.

(C) 2007 British Contact Lens Association. Published by Elsevier Ltd. All rights reserved.
\end{abstract}

Keywords: Refractive index; Automated refractometer CLR 12-70; Silicone-hydrogel contact lenses; Conventional hydrogel contact lens; Equilibrium water content

\section{Introduction}

Refractive index is a physical parameter that reflects the polymers composition of the contact lenses and also their equilibrium water content (EWC), and so it is an important parameter for the optical and physiological perspective. The EWC represents the ability of the hydrogel materials to bind water and it is an important property for clinical behaviour of the contact lenses.

Water plays a key role in the functionality of hydrogel contact lens materials because it has an important effect on the ion and gaseous permeability [1], mechanical and

\footnotetext{
* Corresponding author at: Department of Physics, University of Minho, Campus of Gualtar, Braga, Portugal. Tel.: +351 253604068; fax: +351 253678981 .

E-mail address: mlira@ fisica.uminho.pt (M. Lira).
}

surface properties [2], and also on the biocompatibility of the contact lenses. It has been shown that an increase in refractive index compared to the "true" refractive index is a surrogate outcome for lens dehydration [3]. Lens dehydration can cause changes in contact lenses parameters leading to a decrease in their clinical performance [4-6]. It has also been shown that environmental conditions can significantly affect contact lenses dehydration [4,7-9]. Many studies link contact lens wear discontinuation results to dehydration of the ocular surface, which is one of the main factors restricting the growth of the contact lenses market $[10,11]$.

Although, the evidence is inconclusive that the loss of lens water content occurring during contact lens wear is responsible for dryness symptoms [12-14], the lens materials capacity to maintain its EWC during wear may be an important factor to consider for the clinical success of contact lenses. In fact, clinical and experimental 
observations made in conventional hydrogel contact lenses with higher water content show that these lenses tend to dehydrate on the eye faster and by a greater amount $[12,13,15]$ and some lenses exhibit an irreversible loss of water over time [12].

Patients using frequent replacement lenses complain less of dryness than wearers of other lens types [16,17]. This may be due to increased wettability [18], reduction deposition [19] or reduced dehydration [20]. In addition, wearers of silicone-hydrogel contact lenses are also less aware of lensinduced dryness, particularly at the end of the day [20].

The water content of conventional hydrogel contact lenses is usually above $38 \%$, which contributes to the softness and comfort of these lenses. However, the oxygen permeability is limited by the water phase restricting their wearing schedule. The silicone-hydrogel contact lenses were developed in order to increase the oxygen transmissibility to the cornea, and thus preventing complications due to corneal anoxia observed with the use of conventional hydrogel contact lenses.

Silicone-hydrogel contact lenses are slightly stiffer and have relatively lower water content than the conventional hydrogel materials. The silicone components combined with conventional hydrogel monomers in contact lens materials, increases oxygen permeability but decreases its hydrophilicity [21] making lens surface more hydrophobic and so more prone to deposits adhesion. This has been already established in vitro studies [22-25].

A surface treatment of silicone-hydrogel contact lenses is needed to make them hydrophilic and tolerable on the eye and is an important factor for the clinical performance of these contact lenses. Significant differences exist between the silicone-hydrogels materials $[2,26]$. Balafilcon A, lotrafilcon A and lotrafilcon B are treated using gas plasma techniques, but balafilcon A undergoes plasma oxidation which transforms the silicone components into glassy islands on the surface. Lotrafilcon lenses are treated with hydrocarbon plasma that reacts with air to create continuous hydrophilic surfaces $[2,26]$. On the other hand, galyfilcon A has no surface treatment but incorporates an internal wetting agent that apparently leaches to the lens surface.

Refractive index and EWC are closely linked in conventional soft hydrophilic materials [27,28]. In a previous study was also reported a similar, but independent relationship to that of the conventional hydrogels, between refractive index and EWC for the four silicone-hydrogel contact lenses used in this work [29].

Recently, the automated refractometer CLR 12-70 designed to measure the refractive index of hydrogel lenses has become available. Nichols and Berntsen used this instrument and found it easy to use and it is a reliable and valid technique to determine the refractive index of soft contact lenses [3]. These authors found good reliability within and between operators in the measurements of the refractive index using this refractometer and also demonstrated that this instrument had excellent within-operator reliability. We consider these factors as an advantage of measuring hydrogel refractive index rather than measuring water content directly.

As the refractive index reflects changes in the EWC of the contact lens materials, changes on refractive index, allows the evaluation of the ability of the contact lenses to reach or to maintain their EWC after being worn.

The purpose of this work was to evaluate the ability of four silicone-hydrogel contact lenses (galyfilcon A, balafilcon A, lotrafilcon A and lotrafilcon B) to reach or retain their EWC, through measurements of refractive index, and compare with those occurred on a conventional disposable hydrogel contact lens (etafilcon A).

\section{Material and methods}

A total of 115 commercial lenses were measured: 22 Acuvue $^{\circledR}$ Advance $^{\mathrm{TM}}, 20$ Purevision $^{\mathrm{TM}}, 24$ Focus $^{\circledR}$ Night\&Day ${ }^{\mathrm{TM}}, 19 \mathrm{O}_{2} \mathrm{Optix}^{\mathrm{TM}}$, and 30 Acuvue $^{\mathrm{R}}$. The properties of the contact lens used in this study are detailed in Table 1.

Table 1

Conventional and silicone-hydrogel contact lenses used in this study

\begin{tabular}{|c|c|c|c|c|c|}
\hline Brand & Acuvue $^{\circledR}$ Advance $^{\mathrm{TM}}$ & Purevision $^{\mathrm{TM}}$ & $\begin{array}{l}\text { Focus }^{\circledR} \\
\text { Night } \& \text { Day }^{\mathrm{TM}}\end{array}$ & $\mathrm{O}_{2} \mathrm{Optix}^{\mathrm{TM}}$ & Acuvue $^{\circledR}$ \\
\hline Manufacturer & Johnson \& Johnson & Bausch \& Lomb & CIBA Vision & CIBA Vision & Johnson \& Johnson \\
\hline USAN & Galyfilcon A & Balafilcon A & Lotrafilcon A & Lotrafilcon B & Etafilcon A \\
\hline FDA group & $\mathrm{I}$ & III & I & I & IV \\
\hline Water content $(\%)$ & 47 & 36 & 24 & 33 & 58 \\
\hline Surface treatment & No surface treatment & Gas plasma oxidation & Plasma coating & Plasma coating & No surface treatment \\
\hline RI & $1.4055^{\mathrm{a}}$ & $1.426^{\mathrm{a}}$ & $1.43^{\mathrm{a}}$ & $1.42^{\mathrm{a}}$ & $1.4055^{\mathrm{a}}$ \\
\hline Principal monomers & $\begin{array}{l}\text { mPDMS + DMA + EGDMA + HEMA } \\
+ \text { siloxane macromer + PVP } \\
\text { + visibility tint + UV blocker }\end{array}$ & $\begin{array}{l}\mathrm{NVP}+\mathrm{TPVC}+\mathrm{NCVE} \\
+\mathrm{PBVC}\end{array}$ & $\begin{array}{l}\text { DMA + TRIS } \\
+ \text { siloxane } \\
\text { macromer }\end{array}$ & $\begin{array}{l}\text { DMA + TRIS } \\
+ \text { siloxane macromer } \\
\text { + visibility tint }\end{array}$ & HEMA + MA \\
\hline
\end{tabular}

USAN, United States Adopted Names.

a Obtained from Food and Drug Administration, DMA $N, N$-dimethylacrylamide; PDMS, polydimethylsiloxane; EGDMA, ethylene glycol dimethacrylate; HEMA, poly-2-hydroxiethylmethacrylate; MA, methacrylic acid; NVP, $N$-vinyl pyrrolidone; TPVC, tris-(trimethylsiloxysilyl) propylvinyl carbamate; NCVE, $N$-carboxyvinyl ester; PBVC, poly[dimethysiloxy] di(silylbutanol) bis[vinyl carbamate]. 
The lenses were worn by 58 patients in a daily wear schedule (12-14 h a day) for 30 days in the case of siliconehydrogel. Due to safety reasons, the wearing period of the conventional hydrogel was only 15 days. According to the manufacturer's instructions, galyfilcon A should only be worn for a 15-day period. However, in this study, patients wore this type of lens for 30 days, in order to make possible the comparison with the other silicone-hydrogel contact lenses tested. The average age of patients was $23.3 \pm 6.1$ years (range 16-47 years). The subjects were informed of the proposed procedure and signed a consent form in accordance with the Helsinki Declaration. The criteria for excluding patients from this study were symptoms of dry eye, active ocular illness, systemic disease and any surgery of anterior segment of the eye. All patients used the same multipurpose solution, Renu Multi-Plus ${ }^{\mathrm{TM}}$ (Bausch \& Lomb) to store the lenses overnight. After the trial period, lenses were removed from the patient's eyes and placed in a preservative-free saline solution $(0.9 \% \mathrm{NaCl}$ prepared in distilled water) at least $48 \mathrm{~h}$ before being measured in order to equilibrate and allow the contact lenses to reach their initial EWC.

The samples were measured at a room temperature of $21{ }^{\circ} \mathrm{C}\left( \pm 2{ }^{\circ} \mathrm{C}\right)$ and relative humidity of $52( \pm 2 \%)$. It must be stressed that the solutions used to equilibrate the lenses before measurements do not affect the refractive index values. This was supported by assessing the refractive index in lenses equilibrated in saline solution $(0.9 \% \mathrm{NaCl}$ prepared in distilled water) and the solution contained in the blisters packs (data not shown). The refractive index considered for each lens was the average of five consecutive measurements and each lens was equilibrated and rehydrated between each consecutive measurement by immersion in saline $(0.9 \% \mathrm{NaCl}$ prepared in distilled water).

The refractive indices of all contact lenses were measured via refractometry, then worn, removed, equilibrated and then measured again. The initial value of the lenses refractive index was the value measured after removal from the original pack.

The CLR 12-70 digital automated refractometer (Index Instruments, Cambridge, UK) was used to directly measure the refractive index of all contact lens. This instrument measures refractive index by back reflection at $589 \mathrm{~nm}$ and provides direct refractive index reading with minimal influence of operators subjectivity displaying accurate and reproducible values of refractive index of contact lenses $[3,29]$. The instrument was programmed to use a "continuous scan", and its zero setting was checked before each initial use. The values of refractive index were taken immediately when stability was reached.

The EWC of all contact lenses was obtained using a polynomial relationship between refractive index, obtained directly from CLR 12-70 automated refractometer) [30]

$$
\mathrm{EWC}=952.85 x \mathrm{RI}^{2}-3193.9 x \mathrm{RI}+2664
$$

Using this equation we have the advantage of being able to obtain water content values (clinically important) from refractive index of the lenses obtained using the accurate, rapid, and objective automatic CLR 12-70 refractometer. Without such equation we only can compare refractive index values which are more difficult to interpret in clinical terms. Very small changes in refractive index are able to have significant effects in relative percentage of water content information on the contact lens material.

The change in lens water content of a contact lens can be obtained through the following equation based on Brennan et al. definitions [31]

$\% \Delta \mathrm{EWC}=\frac{\mathrm{EWC}_{\mathrm{w}}-\mathrm{EWC}_{\mathrm{un}}}{\mathrm{EWC}_{\mathrm{un}}} \times 100$

$\mathrm{EWC}_{\mathrm{un}}$ and $\mathrm{EWC}_{\mathrm{w}}$ are the equilibrium water content of the contact lenses before and after wear obtained using Eq. (1).

\subsection{Statistical analyses}

All data were analysed using Statistical Package for Social Sciences, SPSS Version 11.

The differences between refractive index for unworn and worn contact lenses were compared using the paired sample $T$-test for parametric data. For all hypotheses testing, $p \leq 0.05$ was considered statistically significant.

\section{Results}

Table 2 shows the minimum, maximum and average value of the refractive index measured on unworn and worn contact lens.

The values of the refractive index of unworn lenses, determined by automated refractometer, were slightly lower than the nominal values given by Food and Drug Administration (FDA) for the etafilcon A and balafilcon $\mathrm{A}$ lenses and almost the same for galyfilcon $\mathrm{A}$, lotrafilcon $\mathrm{A}$ and lotrafilcon B.

The refractive index of worn silicone-hydrogel contact lenses was not significantly different from that of unworn contact lenses. However, the conventional hydrogel exhibited a significant increase $(p<0.001)$, in the refractive index after being worn (Fig. 1).

Using Eq. (1), we were able to calculate the EWC for each lens used in this work, before and after being worn, and obtain information about the change in EWC of the contact lenses by using Eq. (2). Etafilcon A showed a change in EWC of $10 \%$, and lotrafilcon A of $3 \%$. For galyfilcon A, balafilcon A and lotrafilcon B, the changes in EWC are, respectively $0.9,1$ and $0 \%$. The conventional hydrogel presents higher changes than the silicone-hydrogel contact lens. 
Table 2

Results from refractive index measured in unworn and worn contact lenses

\begin{tabular}{|c|c|c|c|c|c|c|}
\hline & & No. & Minimum & Maximum & Mean \pm S.D. & Significance $(p)$ \\
\hline \multirow[t]{2}{*}{ Etafilcon A } & $\mathrm{u}$ & 30 & 1.361 & 1.405 & $1.398 \pm 0.008$ & \multirow[t]{2}{*}{0.000} \\
\hline & w & 30 & 1.405 & 1.413 & $1.410 \pm 0.002$ & \\
\hline \multirow[t]{2}{*}{ Galyfilcon A } & $\mathrm{u}$ & 22 & 1.405 & 1.414 & $1.408 \pm 0.002$ & \multirow[t]{2}{*}{0.127} \\
\hline & w & 22 & 1.405 & 1.413 & $1.409 \pm 0.002$ & \\
\hline \multirow[t]{2}{*}{ Balafilcon A } & $\mathrm{u}$ & 20 & 1.417 & 1.424 & $1.421 \pm 0.002$ & \multirow[t]{2}{*}{0.467} \\
\hline & w & 20 & 1.418 & 1.425 & $1.422 \pm 0.002$ & \\
\hline \multirow[t]{2}{*}{ Lotrafilcon A } & $\mathrm{u}$ & 24 & 1.425 & 1.428 & $1.426 \pm 0.001$ & \multirow[t]{2}{*}{0.319} \\
\hline & w & 24 & 1.425 & 1.487 & $1.429 \pm 0.124$ & \\
\hline \multirow[t]{2}{*}{ Lotrafilcon B } & $\mathrm{u}$ & 19 & 1.421 & 1.423 & $1.422 \pm 0.008$ & \multirow[t]{2}{*}{0.500} \\
\hline & w & 19 & 1.419 & 1.423 & $1.421 \pm 0.001$ & \\
\hline
\end{tabular}

u: unworn CL; w: worn CL.

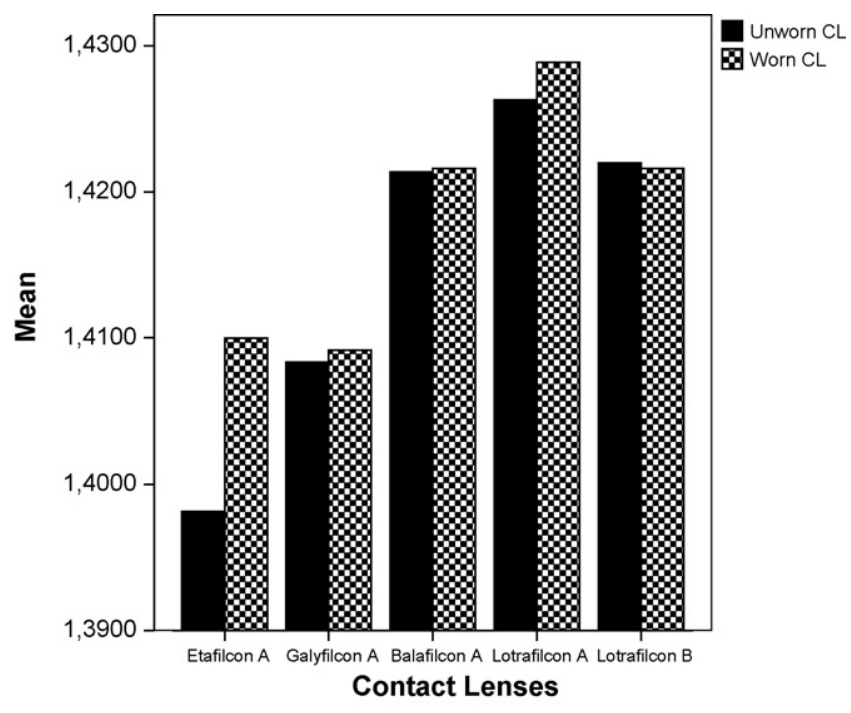

Fig. 1. Mean refractive index for unworn and worn contact lenses.

\section{Discussion}

Water content can be obtained by several methods which include gravimetry [32,33], nuclear magnetic resonance imaging and refractometry. Although the gravimetric technique is more accurate, it is a time-consuming method. In previous work, it has been shown that the values of water content could be obtained from the refractive index using polynomials relationships and the water content and refractive index of conventional hydrogel contact lenses obtained with manual and automated refractometry were comparable [30].

Hand held refractometers are often used in the food and wine industry for measurement of sugar concentration using the Brix scale. This scale represents the grams of sucrose in $100 \mathrm{~g}$ of sucrose solution. Brennan [27] describes the utility of this instrument in measuring refractive indices of contact lenses. However, because the Brix scale is based on a sucrose-water relation, it can induce errors for hydrogel materials that do not have the same water and refractive index relation as sucrose and water [34]. Due to the lower refractive index of siloxane monomer compared to the monomers used in conventional hydrogels, silicone-hydrogel lenses do not fit to the Brix scales. Refractometers calibrated for such scales give a systematic overestimation of contact lens hydration [3,29].

The refractive index can be obtained more accurately with automated refractometry for silicone-hydrogels thus displaying an excellent/positive agreement with nominal data [29]. However, when the refractive index is converted to water content using the Brix scale, the difference between the nominal and the measured value is statistically significant $[3,29]$. This can be explained by the difference in the refractive index of silicone-hydrogels materials and sucrose. However, if the refractive index is measured before and after contact lenses wear, and these values converted to water content using the Brix scales, it is possible to establish comparisons with a good level of accuracy. For siliconehydrogel lenses we are expecting to obtain higher water content values than they actually have. Despite this 
limitation, the values obtained in this study using the same procedure for initial and final measurements of contact lenses will be comparable against each other using Eq. (1).

In hydrogels, the polymer network has free and bound water and the free water molecules can easily evaporate. In the present study, lenses were allowed to reach their EWC in a saline solution after being worn and before being measured. This procedure allows the replacement of lens free water content and enables the evaluation of the ability of the contact lens material to restore the same level of initial water content.

It has been demonstrated that the Acuvue ${ }^{\circledR}$ disposable contact lenses looses significant water content during openeye wear [9]. This loss was considerably greater than that found in other contact lenses, apparently because the polymers are ionic. Several studies have demonstrated that hydrogel contact lenses can suffer in vivo dehydration [35,36] causing a modification in oxygen permeability [33]. It seems that the lens material undergoes chemical changes which can result in variations in the components of the lenses [31]. The fact that silicone-hydrogel contact lenses suffer less dehydration has already been established by in vitro studies [37]. However, because only one side of the lens is exposed to the atmosphere in vivo and because the lipid layer of the tear film may limit lens dehydration, in vitro studies do not exactly mimic real conditions. This makes in vivo studies essential. The capacity of water to diffuse through an hydrogel and to be lost at the lens surface has been suggested to be related to the proportion of relatively loosely bound water, which is greater in a higher water content lens [38]. Evaporative water loss at the anterior lens surface is a potential cause for contact lens dehydration and surface treatment must be considered. The degree and rate of dehydration is related to the material of the lenses and may affect oxygen transmissibility in hydrogel contact lenses [39]. A solution-diffusion water transport model has been developed, which predicts that a soft contact lens on the eye looses water fast until it reaches a steady state [40] and this is consistent with available measurements of contact lens dehydration.

In vitro dehydration studies of conventional and siliconehydrogel contact lenses indicate that evaporation rates from materials are mainly related with water content [41]. Even for lenses belonging to the same FDA group, dehydration behaves differently due to the different material properties [42].

Lens dehydration can have serious implications in lens performance. According to Tranoudis and Efron [43], lens parameters and their properties alter with changes in hydration, and consequently have a decrease in comfort associated to dehydration [44]. So, the ability to contact lens material reach their EWC is a very important fact to consider for the point of view of clinical performance of the contact lens. Morgan and Efron [45] find that some changes occur in water content on hydrogel lenses as a result of ageing and can be related to lens spoilation over time. Also, in another study Morgan and Efron [33] attributed the loss of water content to displacement of water by deposits such as proteins and lipids. Additional related experiments were also conducted to help explain the mechanisms that can lead to contact lens surface damage and formation of deposits [46] and it was proved that silicone-hydrogel contact lenses adsorb a lower amount of proteins than the conventional hydrogel lens [46]. Based on these studies, this can be one possible explanation for the findings presented in the present study.

The present results show that although the different composition, different nominal equilibrium water content $\left(\mathrm{EWC}_{\mathrm{nom}}\right)$ and the different surface properties of the four silicone-hydrogel contact lenses used in this work, the changes in refractive index and the EWCs, before and after being worn, are not statistically significant. These materials appeared to maintain a constant level beyond which its equilibrium water content would not fall.

\section{Conclusions}

The results presented here show that after being worn the silicone-hydrogel contact lens, show more capacity to retain or to reach their initial EWC than conventional hydrogel contact lenses. Since the refractive index is function of water content, the results of this study suggest that the presence of the hydrophobic organosilicon moieties in the siliconehydrogels, their lower EWC and the lower levels of proteins exhibited [46], compared to the conventional hydrogel lens, are the cause of apparent better water retention.

This also suggests that the silicone-hydrogel contact lenses are less susceptible to spoilation over time maintaining its biocompatibility and contributing to the clinical success of lens performance.

\section{Acknowledgments}

The authors have no commercial interests in any of the contact lenses and material used in this paper. We wish to thank Bausch \& Lomb, Ciba Vision and Johnson \& Johnson for donating the lenses used in this work.

\section{References}

[1] Nicolson PC, Vogt J. Soft contact lens polymers: an evolution. Biomaterials 2001;22:3273-83.

[2] Tighe B. In: Sweeney D, editor. Silicone hydrogels: the rebirth of continuous wear. Oxford: Butterworth-Heinemann; 2000. p. 1-21.

[3] Nichols JJ, Berntsen DA. The assessment of automated measures of hydrogel contact lens refractive index. Ophthal Physiol Opt 2003; 23:517-25.

[4] Andrasko G, Schoessler JP. The effect of humidity on dehydration of soft contact lens on the eye. ICLC 1980;7:210-3.

[5] Lowther GE. Lens dehydration: what are the problems and the management? ICLC 1983;10:7.

[6] Paugh JR. Equivalent oxygen percentage as a function of hydration in hydrogel lenses: an in vivo study. Optom Vis Sci 1992;69:805-10. 
[7] Brennan NA, Efron N, Bruce AS, Duldig DI, Russo NJ. Dehydration of hydrogel lenses: environmental influences during normal wear. Am J Optom Physiol Opt 1988;65:277-81.

[8] Morgan PB, Efron N, Morgan SL, Little SA. Hydrogel contact lens dehydration in controlled environmental conditions. Eye Cont Lens 2004;30:99-102.

[9] Cabrera JV, Velasco MJ. Recovery of the water content of hydrogel contact lenses after use. Ophthal Physiol Opt 2005;25:452-7.

[10] Pritchard N, Fonn D, Brazeau D. Discontinuation of contact lens wear: a survey. Int Cont Lens Clin 1999;26:157-62.

[11] Young G, Veys J, Pritchard N, Coleman S. A multi-centre study of lapsed contact lens wearers. Ophthal Physiol Opt 2002;22:516-27.

[12] Efron N, Brennan NA, Bruce AS, Duldig DI, Russo NJ. Dehydration of hydrogel lenses under normal wearing conditions. CLAO J 1987; 13:152-6.

[13] Pritchard N, Fonn D. Dehydration, lens movement and dryness ratings of hydrogel contact lenses. Ophthal Physiol Opt 1995;15:281-6.

[14] Nichols JJ, Sinnott LT. Tear film, contact lens, and patient-related factors associated with contact lens-related dry eye. Invest Ophthal Vis Sci 2006;47:1319-28.

[15] Andrasko G. Hydrogel dehydration in various environments. ICLC 1983;10:23-8.

[16] Nason RJ, Boshnick EL, Cannon WM. Multisite comparison of contact lens modalities. Daily disposable wear vs. conventional daily wear in successful contact lens wearers. J Am Optom Assoc 1994; 65:774-80.

[17] Boswall GJ, Ehlers WH, Luistro A, Worrall M, Donshik PC. A comparison of conventional and disposable extended wear contact lenses. CLAO J 1993;19:158-65.

[18] Cheng L, Muller SJ, Radke CJ. Wettability of silicone-hydrogel contact lenses in the presence of tear-film components. Curr Eye Res 2004;28:93-108.

[19] Jones L, Franklin V, Evans K, Sariri R, Tighe B. Spoilation and clinical performance of monthly vs. three monthly Group II disposable contact lenses. Optom Vis Sci 1996;73:16-21.

[20] Fonn D, Pritchard N, Dumbleton K. In: Sweeney D, editor. Silicone hydrogels: the rebirth of continuous wear contact lenses. Oxford, UK: Butterwrth-Heinemann; 2000. p. 214-34.

[21] Tighe B. Silicone hydrogels: what are they and how should they be used in everyday practice? Optician 1999;31-2.

[22] Jones L, Senchyna M, Glasier MA, Schickler J, Forbes I, Louie D, et al. Lysozyme and lipid deposition on silicone hydrogel contact lens materials. Eye Con Lens 2003;29:S75-9.

[23] Moradi O, Modarress H, Noroozi M. Experimental study of albumin and lysozyme adsorption onto acrylic acid (AA) and 2-hydroxyethyl methacrylate (HEMA) surfaces. J Colloid Interface Sci 2004;271:16-9.

[24] Bruinsma GM, van der Mei HC, Busscher HJ. Bacterial adhesion to surface hydrophilic and hydrophobic contact lenses. Biomaterials 2001;22:3217-24.

[25] Henriques M, Sousa C, Lira M, Real Oliveira MECD, Oliveira R, Azeredo J. Adhesion of Pseudomonas aeruginosa and Staphylococcus epidermidis to silicone-hydrogel contact lenses. Optom Vis Sci 2005;82:446-50.

[26] Lopez-Alemany A, Compan V, Refojo MF. Porous structure of Purevision versus Focus Night\&Day and conventional hydrogel contact lenses. J Biomed Mater Res 2002;63:319-25.
[27] Brennan NA. A simple instrument for measuring the water content of hydrogel lenses. ICLC 1983;357-61.

[28] Mousa GY, Calander MG, Sivak JG, Egan DJ. The effect of the hydration characteristics of hydrogel lenses on the refractive index. ICLC 1983;31-7.

[29] Gonzalez-Meijome JM, Lira M, Lopez-Alemany A, Almeida JB, Parafita MA, Refojo MF. Refractive index and equilibrium water content of conventional and silicone hydrogel contact lenses. Ophthal Physiol Opt 2006;26:57-64.

[30] Gonzalez-Meijome JM, Lopez-Alemany A, Lira M, Almeida JB, Real Oliveira MECD, Parafita MA. Equivalences between refractive index and equilibrium water content of conventional and silicone hydrogel soft contact lenses from automated and manual refractometry. J Biomed Mater Res B Appl Biomater 2007;80:184-91.

[31] Brennan NA, Efron N, Truong VT, Watkins RD. Definitions for hydration changes of hydrogel lenses. Ophthal Physiol Opt 1986;6:333-8.

[32] Alemany AL, Refojo MF. Comparative study of the hydration of hydrophilic contact lenses by refractive index and gravimetry. CLAO J 2000;26:200-3.

[33] Morgan PB, Efron N. In vivo dehydration of silicone hydrogel contact lenses. Eye Cont Lens 2003;29:173-6.

[34] Nichols JJ, Mitchell GL, Good GW. The reliability and validity of hand-held refractometry water content measures of hydrogel lenses. Optom Vis Sci 2003;80:447-53.

[35] Brennan NA, Lowe R, Efron N, Harris MG. In vivo dehydration of disposable (Acuvue) contact lenses. Optom Vis Sci 1990;67: 201-3.

[36] Fonn D, Situ P, Simpson T. Hydrogel lens dehydration and subjective comfort and dryness ratings in symptomatic and asymptomatic contact lens wearers. Optom Vis Sci 1999;76:700-4.

[37] Kohler JE, Flanagan GW. Clinical dehydration of extended wear lenses. Int Cont Lens Clin 1985;152-61.

[38] Tranoudis I, Efron N. Water properties of soft contact lens materials. Cont Lens Anterior Eye 2004;27:193-208.

[39] Efron N, Morgan PB. Hydrogel contact lens dehydration and oxygen transmissibility. CLAO J 1999;25:148-51.

[40] Fornasiero F, Prausnitz JM, Radke CJ. Post-lens tear film depletion due to evaporative dehydration of a soft lens. J Membr Sci 2006;229-43.

[41] Jones L, May C, Nazar L, Simpson T. In vitro evaluation of the dehydration characteristics of silicone hydrogel and conventional hydrogel contact lens materials. Cont Lens Anterior Eye 2002; 25:147-56.

[42] Hall B, Jones S, Young G, Coleman S. The on-eye dehydration of proclear compatibles lenses. CLAO J 1999;25:233-7.

[43] Tranoudis I, Efron N. Parameter stability of soft contact lenses made from different materials. Cont Lens Anterior Eye 2004; 27:115-31.

[44] Lebow K, Bridgewater B. A three-month comparative daily-wear study of two high water content soft lenses. ICLC 1997;198-205.

[45] Morgan PB, Efron N. Hydrogel contact lens ageing. CLAO J 2000;26:85-90.

[46] Santos L, Rodrigues D, Lira M, Real Oliveira MECD, Oliveira R, Azeredo J. The influence of surface treatment on hydrophobicity, protein adsorption and microbial colonisation of silicone hydrogel contact lenses. Cont Lens Anterior Eye 2007;30:183-8. 